\title{
A PRÁTICA DA REPLICAÇÃO EM PESQUISAS DO TIPO SURVEY EM ADMINISTRAÇÃO DE EMPRESAS
}

\author{
THE REPLICATION OF SURVEY-BASED RESEARCH IN \\ BUSINESS ADMINISTRATION
}

Recebido em: 31/10/2012 Aprovado em: 20/01/2013 Avaliado pelo sistema double blind review Editora Científica: Manolita Correia Lima

\section{MARIA LAURA FERRANTY MAC LENNAN ferranty@hotmail.com ILAN AVRICHIR \\ ESCOLA SUPERIOR DE PROPAGANDA E MARKETING}

\begin{abstract}
RESUMO
A replicação de pesquisas do tipo survey nas ciências sociais aplicadas é amplamente encorajada pela teoria, por sua ligação com os avanços da ciência. O presente ensaio visa discutir o seu significado, a validade da repetição de pesquisas e sua incidência no Brasil. Além disso, algumas tipologias descritas na literatura são analisadas. Abordam-se a meta-análise e o seu relacionamento com replicações, as dificuldades e reflete-se sobre o porquê de se promover e publicar esse tipo de estudo acadêmico. Como fonte de evidências, o presente estudo analisa a incidência de pesquisas com a utilização da prática em amostra de publicações científicas nacionais na área de administração e observa a sua baixa ocorrência. Adicionalmente, incluem-se sugestões, de modo a incentivar a utilização da replicação, especialmente dirigida aos editores, pesquisadores e estudantes de Administração. Palavras-chave: replicação; survey; pesquisa em administração; meta-análise.
\end{abstract}

\footnotetext{
ABSTRACT

Replicating surveys in applied social sciences is widely encouraged in theory due to its contributions to science. This paper aims to discuss the significance of this, the validity of conducting previous research using replicated surveys and its incidence in Brazil. Furthermore, certain typologies described in the literature are explained, and metaanalysis and how it relates to replication is touched on. The paper also discusses the difficulties and reflects on why this kind of academic study is promoted and published. The paper examines Brazilian scientific publications in the Business Administration area for incidences of replication, finding that relatively few replications studies have been published. Finally, we propose means to encourage their use, especially directed to editors, researchers and Business Administration students.

Keywords: replication; survey; business administration research; meta-analysis.
} 


\section{INTRODUÇÃO}

Este ensaio busca cooperar com a compreensão do significado da prática de replicação nas pesquisas realizadas com base em surveys e de sua contribuição para o progresso do conhecimento científico.

Com o aumento da diversidade de tecnologias e a ampliação do número de estudos desenvolvidos no país, a replicação de pesquisas torna-se uma forma eficaz de se checar as teorias e achados científicos (MORRISON; MATUSZEK; SELF, 2OIO).

Apesar de existirem diversos processos de pesquisa científica, o rigor metodológico, a coerência sistemática no processo de coleta e análise dos dados e a definição de um objeto de investigação são bases comuns às várias atividades de investigação (COLLIS; HUSSEY, 1997). A pesquisa pode ser conceituada como um processo metodológico de busca pelo conhecimento, dito processo epistemológico. Da mesma forma que "epistemologia presume metodologia, esta presume replicabilidade" (BERTHON et al., 2002, p.4I6).

Ao se repetir um levantamento de dados do tipo survey, buscase comparar dados e encontrar resultados similares aos originais (EVANSCHITZKy et al., 2007; BerThon et al., 2002). Deste modo, replicar significa algo vital para o progresso científico. No entanto, a replicação de uma pesquisa é considerada uma prática bastante rara (BURMAN; REEd; ALM, 20IO; EVANSCHITZKY; ARMSTRONG, 2009; TSAng; KWAn, I999; HUBBARd; VETTER; LITTLE, I998).

Vale ressaltar, antes de tudo, que o autor pioneiro deve entender a reprodução de sua pesquisa como a mais sincera homenagem ao seu trabalho (TENóRIO, 2009).

O presente trabalho toma como ponto de partida evidências, advindas de consultas bibliométricas às principais revistas nacionais, de que não se verifica a utilização da replicação como prática de investigação em Administração de Empresas nas principais publicações nacionais. Aqui será discutido o porquê de existirem tão poucos artigos com a utilização 
desta prática e quais os incentivos e desincentivos para a condução de duplicações e extensões de pesquisas. Além de discutidas estas temáticas, serão encaminhadas algumas sugestões.

Neste ensaio, delimita-se a argumentação sobre replicação de levantamento de dados do tipo survey. Este é compreendido como um dos diversos processos investigativos possíveis na pesquisa quantitativa (ALRECK; SETTLE, 2004) e pode ser caracterizado pela manipulação de dados e pela consequente mensuração dos resultados com base na experimentação e na observação dos fenômenos. De acordo com Cooper e Schindler (2008), survey é um processo de mensuração apropriado para o levantamento de informações em uma entrevista altamente estruturada, com ou sem o envolvimento do entrevistador. Geralmente utiliza-se a metodologia quantitativa, a qual, no estudo de uma determinada população, por exemplo, possibilita a inferência de conclusões sobre ela, por meio de observações de uma pequena amostra desta população (COLLIS; HUSSEY, 1997).

A replicação de pesquisas qualitativas não foi abordada no presente ensaio. Esta escolha se deve às características epistemológicas e ontológicas que diferenciam o método qualitativo do quantitativo. Na pesquisa qualitativa o pesquisador interage diretamente com o objeto de pesquisa (CRESWELL, 1994). Já na pesquisa quantitativa o pesquisador se posiciona de modo independente em relação ao seu objeto de investigação. Ontologicamente, no método qualitativo a realidade é considerada subjetiva e múltipla de acordo com a visão dos participantes do estudo. Entretanto, no caso da pesquisa quantitativa, a realidade é considerada objetiva e singular, independente do pesquisador (CRESWELL, 1994). O método qualitativo possui critérios de verificação distintos dos aplicados na pesquisa quantitativa e a replicação do método é impactada pelos aspectos epistemológicos e ontológicos expostos. Por esses motivos se justifica o recorte escolhido.

\section{O QUE É E O QUE NÃO É REPLICAÇÃO}

A literatura sobre replicação apresenta poucas divergências sobre a sua definição. Replicar significa pesquisar novamente com a finalidade de 
observar, investigar, experimentar, comparar os resultados, validar e definir claramente as teorias (MORRISON; MATUSZEK; SELF, 2OIO; RIEDL, 2007; BERTHON et al., 2002). Dada a definição do conceito, examinam-se as suas aplicações.

Tradicionalmente, a duplicação de um survey é realizada de modo a determinar se as descobertas deste estudo são reproduzíveis (SINGH; ANG; LEONG, 2003; TSANG; KWAN, 1999). Hubbard, Vetter e Little (1998) afirmam que a replicação pode consolidar a generalização dos resultados da pesquisa original. Como consequência, os cientistas sociais poderiam alicerçar o conhecimento em fundamentos sólidos e fazer significativos progressos, pois as descobertas seriam específicas e reproduzíveis (MORRISON; MATUSZEK; SELF, 20IO).

De acordo com os autores, a replicação com extensão é a duplicação de um estudo previamente publicado, com o objetivo de expandir a validade externa, ou generalizar seus resultados (EVANSCHITZKY et al., 2007; TONCAR; MUNCH, 2OIO; HUBBARD; VETTER; LITTLE, 1998). É reconhecidamente importante delimitar sob que condições as descobertas se mantêm. Esta delimitação pode ser conduzida de diversas formas, e uma possibilidade seria, baseada no exemplo dado acima, replicar o mesmo estudo em outra amostra da mesma população (HUBbARD; VETTER; LITTLE, 1998).

Para que a generalização dos resultados de uma pesquisa possa ocorrer, é necessário repetir o estudo original, como a suposta pesquisa sobre uma população, em uma população diferente (TSANG; KWAN, I999). Os procedimentos da pesquisa mãe devem ser seguidos rigorosamente, de modo a garantir a comparabilidade dos resultados.

A extensão da nova pesquisa poderia modificar o desenho do estudo original, como na definição das variáveis dependentes e independentes. Isso se justificaria pelo objetivo de averiguar se os resultados prévios são válidos e podem ser extrapolados para outras populações, categorias de produtos, períodos de tempo, novas localizações geográficas ou se são somente idiossincrasias ou peculiaridades localizadas (HUBBARD; VETTER; LITTLE, I998).

O tempo inevitavelmente altera o sujeito e o objeto, o pesquisador e o campo a ser investigado. A replicação absoluta é um ideal muitas vezes 
inalcançável no campo das ciências sociais. Por um lado, pode-se replicar o problema original, embasamento teórico e metodologia, por outro lado, é possível gerar novos conhecimentos em termos do problema, método utilizado, desenvolvimento teórico e contexto da pesquisa prévia (BERTHON et al., 2002).

Os autores Easley, Madden e Dunn (2000) propõem quatro tipologias para a replicação, mas apenas três são aplicáveis às ciências sociais: Replicação tipo I - cópia fiel da pesquisa original; Replicação tipo II - cópia aproximada da pesquisa original; Replicação tipo III - alteração deliberada da pesquisa original. De acordo com os autores, o propósito da Replicação do tipo I é reproduzir o estudo original da forma mais fidedigna possível. $\mathrm{Na}$ Replicação do tipo II busca-se repetir de modo aproximado a pesquisa original com a alteração controlada de um aspecto da pesquisa, como, por exemplo, testar o mesmo fenômeno em outro contexto. Já na Replicação do tipo III, os autores da nova pesquisa efetuam modificação deliberada em relação à pesquisa base. Esta alteração pode ser metodológica e/ou conceitual. $\mathrm{O}$ tipo não aplicável às ciências sociais é a repetição precisa, na qual cada nuance da pesquisa original é replicada.

Por sua vez, Morrison, Matuszek e Self (20I0) partem da classificação utilizada por outros autores (DARLEY, 2000; HENDRICK, I99I) e descrevem quatro tipos de replicações: estrita, parcial, operacional e conceitual. A replicação estrita se propõe a repetir um estudo, de modo a buscar aderência máxima ao maior número possível de aspectos desse estudo. A replicação parcial introduz alterações em um aspecto da pesquisa original, com o propósito de ampliar o escopo da pesquisa e sua generalização. A do tipo operacional busca validar os procedimentos de amostragem e a metodologia do experimento original. Finalmente, na replicação conceitual, o pesquisador busca confirmar as hipóteses do estudo original por meio de uma metodologia alternativa àquela já aplicada.

Nas ciências sociais, diferentemente das ciências naturais, a replicação literal não é possível (MADDEN; EASLEY; DUNN, 2005; SINGH; ANG; LEONG, 2003; TSANG; KWAN, 1999). Tal afirmação gera um paradoxo, pois, uma vez que as conclusões originais não são repetidas, o pesquisador pode ser acusado de 
desvio metodológico (TONCAR; MUNCH, 20IO; MADDEN; EASLEY; DUNN, 2005); mas, se o pesquisador confirmar as conclusões originais, o argumento usado será o de que nada novo foi aprendido.

Singh, Ang e Leong (2003) classificam as repetições de pesquisas em seis tipos, de acordo com duas dimensões: (I) uso dos mesmos métodos de medição de constructos e análise de dados e (2) utilização das mesmas fontes de dados. As seis categorias resultantes (conferência da análise, replicação exata, generalização empírica, reanálise de dados, extensão conceitual e generalização e extensão) são úteis para a compreensão das diferentes abordagens de replicações.

As múltiplas tipologias contribuem para o entendimento da visão dos diversos autores sobre o tema. As replicações do tipo I, segundo Easley, Madden e Dunn (2000), ou estrita, de acordo com Morrison, Matuszek e Self (20I0), dependem da disponibilidade dos dados do projeto a ser replicado (HAMERMESH, 2007).

Como este ensaio trata de pesquisas do tipo survey em ciências sociais aplicadas, pressupõe-se que os dados obtidos pelas replicações de levantamentos podem ser diferentes dos do experimento original. Esse tipo de descoberta é útil para conferir robustez às pesquisas e para delimitar até onde constructos podem ser extrapolados (EASLEY; MADDEN; DUNN, 2000).

Especificamente no caso de levantamentos do tipo surveys, o seu valor é maior caso as réplicas permitam que o leitor amplie as inferências e generalizações. Essas pesquisas são baseadas em meta-análises, que, por sua vez, são subsidiadas por estudos replicados de alta qualidade (EDEN, 2002). A replicação é elemento essencial na avaliação e unificação de resultados de um grande grupo de estudos (DEWALD; THURSBY; ANDERSON, 1986).

A meta-análise utiliza ferramentas estatísticas para combinar estudos independentes, voltados a uma questão única, e pode ser entendida como "estudo dos estudos" (MORRIson; MATUSZEK; SELF, 20IO). Estudos sobre objetos semelhantes podem ser comparados mesmo se desenvolvidos por meio de metodologias e amostras diferentes. Isto permite que se chegue a novas conclusões ou que se endosse uma teoria ou constructo teórico, baseado na meta-análise de pesquisas afins. Hunter (200I) aponta a tendência 
de crescimento da utilização da meta-análise como instrumento de averiguação da literatura pré-existente a respeito de uma questão a ser estudada.

A meta-análise não é uma repetição de pesquisas existentes (MORRISON; MATUSZEK; SELF, 20Io), mas, sem a replicação, ela não possui material de análise para contribuir com a construção do conhecimento (EDEN, 2002). A meta-análise é uma ferramenta desenvolvida para comparar resultados entre replicações, mesmo que não se trate de replicações exatas, ou estritamente semelhantes (HUNTER, 200I).

\section{PORQUE REPLICAR É PRECISO}

Não há problema em considerar a criatividade como critério de qualidade de pesquisas científicas, pois o progresso científico depende em parte de novas ideias. Por outro lado, a evolução científica também necessita de fatos que possam ser estabelecidos apenas baseados na replicação de estudos (HUNTER, 200I).

A importância da replicação reside, portanto, em garantir a validade e confiabilidade da correta condução metodológica, com o papel de proteger o leitor contra a aceitação de resultados não criticados (sINGH; ANG; LEONG, 2003; TSANG; KWAN, I999). Pode-se dizer, portanto, que as replicações são instrumentos de validação e de definição da aceitação de teorias (MORRISON; MATUSZEK; SELF, 20Io). Além disso, as replicações de pesquisas podem identificar fenômenos que influenciaram o estudo anterior, como vieses do estudioso pioneiro. Outro benefício potencial da prática da réplica é o de se checarem as questões de pesquisa e as metodologias utilizadas, além do benefício de conferir validade interna e amplificar a generalização dos resultados de uma pesquisa.

Numerosos autores indicaram que a integridade de sua literatura estava comprometida particularmente por erros como a rejeição incorreta da hipótese nula e por outros resultados dúbios (BURMAN; REED; ALM, 2OIO; SINGH; ANG; LEONG, 2003; HUBBARD; VETTER; LITTLE, I998). Hunter (200I) alerta também quanto aos erros na definição correta do tamanho da amostra. 
Os estudos em Administração de Empresas são relativamente recentes, se comparados à Microeconomia ${ }^{1}$, por exemplo. A imaturidade e a natural complexidade dos estudos científicos geram a necessidade da análise e validação dos resultados, e a réplica pode ser uma forma de conferir confiabilidade às hipóteses e conclusões de um estudo (SINGH; ANG; LEONG, 2003).

Repetições podem verificar resultados inesperados ou incomuns e incrementar as descobertas com desdobramentos posteriores (MORRISON; MATUSZEK; SELF, 20IO; TONCAR; MUNCH, 20I0). A replicação checa a acurácia das descobertas, ao estressar a sua validade, sua confiabilidade e a sua objetividade (BERTHON et al., 2002).

O processo de réplica de pesquisas é relevante, pois uma teoria deve atender a dois pré-requisitos: (a) reprodutibilidade - possibilidade de identificar as relações causais em amostra e contexto similar - e (b) teste de generalização - possibilidade de identificar as relações causais em amostra e contexto diferentes (RIEDL, 2007; BERTHON et al., 2002).

King (1995) afirma que, atualmente, o modo mais cientificamente produtivo de construção do conhecimento em pesquisa é a replicação de descobertas realizadas. As replicações de pesquisas que confirmam as hipóteses originais aumentam a confiabilidade da pesquisa original, estabelecem a replicabilidade e fortalecem as iniciativas de triangulações (MORRISON; MATUSZEK; SELF, 2OIO).

Segundo Uncles (20II), a replicação é importante por três razões principais: 1 Possibilita observar se o resultado alcançado no estudo original será alcançado na sua repetição;

2 Estabelece que o resultado geralmente se repete;

3 Estabelece onde o resultado geralmente não se repete.

A replicação tem a finalidade de proteger o leitor contra a aceitação de resultados sem a devida crítica (SINGH; ANG; LEONG, 2003; TSANG; KWAN, I999; KING, 1995). Ela permite a revisão de um estudo por parte de outros pesquisadores e o desenvolvimento do rigor da ciência. Em um campo emergente, como o da Administração de Empresas, replicar é essencial

1 Esta ciência utiliza modelos complexos com variáveis múltiplas e relacionamentos difíceis de isolar. 
(SINGH; ANG; LEONG, 2003; HUBbARD; VETTER; LITTLE, I998). Este princípio é válido para a ciência em geral e pode ser aplicado em todas as áreas do conhecimento (MORRISON; MATUSZEK; SELF, 2OIO).

Finalmente, repetir o experimento para estabelecer a validade externa da relação causal do estudo é a aspiração da réplica, de forma a contribuir para o refinamento de uma teoria (EASLEY; MADDEN; DUNN, 2000). Afinal, a reprodução da metodologia suporta a integridade das relações causais obtidas na pesquisa original.

Nas ciências sociais, a correta documentação metodológica é necessária, caso se deseje duplicar um estudo (KING, I995). Em um projeto de pesquisa é necessário descrever os métodos de amostragem e de coleta de dados, além das técnicas de mensuração aplicadas (VELUDO-DE-OLIVEIRA; OLIVEIRA, 20I2). Informações como a descrição das fases da pesquisa frequentemente não são de domínio público e, para se refazer a etapa de levantamento dos dados, é preciso contar com a colaboração do autor; colaboração esta que não pode ser garantida ao autor da réplica.

"Se um estudo não pode ser reproduzido, qual o propósito do mesmo?" King (I995, p. 445) questiona qual seria a proteção dada aos pesquisadores que investem tempo na leitura de trabalhos sem fundamentação metodológica comprovada.

Em consonância com as preocupações já expostas, Bido et al. (20I2) avaliam a qualidade dos registros metodológicos de 68 artigos publicados sobre modelagem de equações estruturais, e uma das principais conclusões dessa análise é a de que "não é possível replicar os estudos devido à falta de informações e o uso de estratégias exploratórias sem a posterior validação" (BIDO et al., 20I2, p. I25).

Independentemente de a duplicação produzir ou não os mesmos resultados da pesquisa original, ela acrescenta informações à investigação. Caso ela "apenas" repita as mesmas descobertas, enriquece os dados relevantes para a teoria testada e aumenta a confiança na hipótese de generalização, além de produzir material para futuras meta-análises (HUNTER, 200I).

Parece consenso o fato de que a decisão de se aceitar ou rejeitar uma hipótese é mais bem fundamentada com base em um grande número de 
dados do que em poucos dados. Se a replicação exata de um estudo produz resultados contraditórios ao original, estes resultados acarretam dúvidas e tornam necessárias novas pesquisas a respeito da questão mãe (EDEN, 2002; EASLEY; MADDEN; DUNN, 2000).

Este tipo de contradição pode transformar conclusões científicas em "folclore gerencial". Isto ocorreu, por exemplo, em trabalho muito citado na área de marketing, de autoria de Pomerance e Zielske (I959), que estuda a eficácia da propaganda concentrada no tempo versus a eficácia da propaganda espaçada. As conclusões publicadas pelos autores foram as de que a propaganda concentrada (conhecida como pulse) é mais efetiva do que a propaganda espaçada, pois produz maiores picos de lembranças das peças publicitárias. Simon (1979), 20 anos depois, obteve os dados brutos originais e verificou que os números apresentavam resultados opostos: a propaganda espaçada no tempo é mais efetiva, financeiramente, do que a propaganda concentrada. A reanálise do artigo original, baseada nos dados brutos, gerou severas críticas ao processo científico, pois foram necessários 20 anos para que a veracidade das conclusões originais fosse contestada. Obviamente, mesmo com a posterior correção advinda da replicação, muitas generalizações foram feitas com base na informação de 1959 (EASLEY; MADDEN; DUNN, 2000).

A despeito de todas as razões citadas acima, a literatura está repleta de estudos originais não replicados ou validados, cuja veracidade permanece intocada e cujas conclusões são disseminadas como leis inquestionáveis (EASLEY; MADDEN; DUNN, 2000). 


\section{A INCIDÊNCIA DE REPLICAÇÃO DE PESQUISAS DO TIPO SURVEY EM ADMINISTRAÇÃO DE EMPRESAS}

Uncles (20II) aponta evidências de que $60 \%$ dos resultados advindos das replicações de pesquisas conflitam com os obtidos nos estudos originais. Mesmo com indícios elevados de divergências em relação aos resultados originais, não se verifica o hábito de se replicarem os levantamentos do tipo survey, a fim de confirmar as suas conclusões.

Em um estudo bibliométrico, Evanschitzky et al. (2007) analisam as replicações publicadas nos seguintes periódicos: Journal of Marketing (JM), Journal of Marketing Research (JMR) e Journal of Consumer Research. Os autores averiguam que, no período de 1990 a 2004, a média de publicações de replicações foi de $1,2 \%$, quando comparada à de publicações de novos estudos. Pesquisa similar em journal alemão (Marketing ZFP) apresenta taxa de publicação de replicações de $0,6 \%$.

O presente trabalho realizou uma análise bibliométrica, que abrange o referencial teórico em Administração de Empresas já publicado nacionalmente, inspirada nos baixos indicadores estrangeiros da prática de replicações. O critério de escolha utilizado para definir quais seriam os periódicos analisados levou em consideração o sistema de avaliação de periódicos QUALIS, da CAPES (20I2). Optou-se por aqueles periódicos classificados como "A" nacional em revistas (CAPPELle et al., 2006).

Por meio dessa análise bibliométrica, verifica-se qual é o indicador de publicação de replicações de levantamentos do tipo surveys, nas principais revistas acadêmicas de estudos em Administração de Empresas, no Brasil. Tal método científico, delineado como documental, por se basear em material disponível ao público em geral, é usado para pesquisar a evolução de um campo científico, investigar suas principais contribuições e o aparecimento de novos temas, entre outras utilizações (MARTignago; ALPERSTEDT, 20II). As buscas foram realizadas no dia 25/03/2012 e abrangeram publicações no período de janeiro de 2002 a janeiro de 2012.

Nas publicações científicas nacionais - quais sejam, BAR: Brazilian Administration Review, RAC: Revista de Administração Contemporânea, 
o\&s: Organizações e Sociedade, RAE: Revista de Administração e Economia, Revista Árvore - não foram encontradas referências às palavras replicação, replicar ou replicate no sentido exposto no presente ensaio.

Na revista RAC, o artigo de Marchetti e Prado (2004) apresenta modelo de avaliação da satisfação do consumidor quanto ao setor elétrico brasileiro e sugere sua replicação em outros setores da economia brasileira. No mesmo periódico o trabalho de Neves e Costa (2008), "Fatores de localização de postos de combustíveis em Fortaleza", aponta que uma das contribuições do estudo reside na sua possibilidade de replicação. Ambos os artigos não aplicam a prática da replicação em seus procedimentos de pesquisa, embora utilizem o termo.

Identificou-se na revista o\&s o uso da palavra "replicar" no trabalho de Bido et al. (2012, p. I25), de título "Qualidade dos Relatos dos Procedimentos Metodológicos em Periódicos Nacionais na Área de Administração de Empresas: o caso da modelagem de equações estruturais nos periódicos nacionais entre 200I e 2010", em que os autores se referiam à "impossibilidade de replicar estudos anteriores". No artigo, mesmo com a presença do termo replicação, a prática não é identificada.

No caso da revista RAE observa-se a incidência do vocábulo replicação no artigo "análise de métodos de replicação: o caso IBOVESPA" em que Sheng e Saito (2002) replicam um índice em uma carteira de investimento chamada "carteira espelho". A replicação nesse caso se trata de instrumento de acompanhamento de retorno de um determinado índice de mercado.

Pode-se afirmar que o sentido exposto no presente ensaio não pode ser verificado nos trabalhos nomeados acima. Os autores não utilizaram a replicação como prática de pesquisa a fim de alcançar objetivos de verificação, generalização e confirmação de teorias ou hipóteses propostas por outros autores e pesquisadores. Portanto, o índice de replicação nas publicações brasileiras citadas acima é zero.

Em quatro periódicos consultados, RAP: Revista de Administração Pública, Cadernos de Saúde Pública, Psicologia: Reflexão e Crítica e Rausp: Revista de Administração da Universidade de São Paulo foram identificados 
artigos com a incidência da palavra "replicar" no sentido abordado no presente ensaio.

Em artigo publicado na RAP, Prates (2009) replicou qualitativamente uma pesquisa quantitativa, no estudo "Redes sociais em comunidades de baixa renda: os efeitos diferenciais dos laços fracos e dos laços fortes". Essa replicação pode ser classificada, segundo a tipologia de Morrison, Matuszek e Self (20I0), como replicação conceitual, pois a metodologia de pesquisa foi alterada em relação ao estudo original.

O trabalho de Moreno et al. (2006), publicado na revista Cadernos de Saúde Pública, replica o Instrumento Abreviado de Avaliação de Qualidade de Vida da Organização Mundial da Saúde. Identifica-se, portanto a utilização da prática nesse artigo.

Na revista Psicologia: Reflexão e Crítica identificaram-se quatro trabalhos com a incidência do vocábulo replicar, todavia apenas dois atendem o sentido do presente ensaio. Trata-se dos artigos (i) "Treino de memória no idoso saudável: benefícios e mecanismos", em que Yassuda et al. (2006) replicam em amostra brasileira teste sobre memória em idosos saudáveis; e (ii) "Influência social, atribuição de causalidade e julgamentos de responsabilidade e justiça”, de Rodrigues e Assmar (2003). No último, os autores replicam achados anteriores e introduzem refinamentos metodológicos

De acordo com Morrison, Matuszek e Self (2010), os trabalhos de Moreno et al. (2006) e de Yassuda et al. (2006) podem ser classificados como replicação parcial. A modificação principal observada nesses casos em relação ao estudo original foi a aplicação do instrumento estrangeiro no contexto brasileiro. Já no caso do artigo de autoria de Rodrigues e Assmar (2003), o mesmo pode ser entendido como replicação conceitual, ao se modificar a metodologia de pesquisa em relação ao estudo original. Neste último caso não se repetiu o survey.

$\mathrm{Na}$ revista RAUSP, tal palavra foi encontrada em duas publicações. No artigo de Neves (2007), "A identidade e a focagem dos empregados de restaurantes de Fortaleza". Este, apesar de citar a palavra replicar, não 
consiste em uma replicação, pois o vocábulo foi utilizado para fazer referência a possíveis desdobramentos futuros. Finalmente na RAUSP, no artigo "Investimentos de capital nas grandes empresas", publicado pelos professores e pesquisadores Fensterseifer e Saul (1993), os autores replicam parcialmente uma pesquisa realizada em 1985, estudando a mesma população da pesquisa anterior, com a intenção de compreender a evolução no comportamento de determinadas empresas. Esse pode ser classificado como replicação parcial ao ser resgatada a tipologia de Morrison, Matuszek e Self (20I0). A modificação principal verificada no estudo reside na análise da evolução temporal entre a pesquisa original e a sua repetição.

Como resultado da pesquisa bibliométrica, em nove dos principais periódicos nacionais, segundo os critérios da CAPES, em amplo período pesquisado, identificaram-se apenas cinco trabalhos que utilizassem a replicação como prática de pesquisa. Com base neste levantamento, comprovou-se que há poucas evidências do uso da prática de replicação em Administração de Empresas no Brasil. Por este motivo, infere-se que pode haver falhas nos processos de verificação, extensão e produção do conhecimento nas pesquisas científicas na área, em decorrência da ausência da prática da replicação. Isto se deve à falta de verificação da integridade das descobertas por meio da repetição dos estudos.

Algumas possibilidades para a escassez de replicações de pesquisas nas produções nacionais podem ser levantadas. Não há uma cultura de replicação, e esta prática é vista como grande demandante de tempo, trabalhosa, incomum, pouco criativa, sem visão e desinteressante (UNCLES, 20II; BERTHON et al., 2002; EASLEY; MADDEN; DUNN, 2000). Na melhor das hipóteses, a replicação é considerada um empreendimento destinado aos pesquisadores menos habilidosos. Repetições de experimentos em ciências sociais aplicadas, especificamente em Administração de Empresas, não se encaixam no paradigma de resolução de problemas de pesquisa, que define a estrutura de recompensas da pesquisa científica (BURMAN; REED; ALM, 20IO; BERTHON et al., 2002; TSANG; KWAN, I999; DEWALD; THURSBY; ANDERSON, I986). A mesma conclusão exposta na literatura estrangeira pode ser aplicada ao contexto da pesquisa nacional em Administração de Empresas. 
Singh, Ang e Leong (2003) argumentam que os motivos para a baixa ocorrência de replicações são: (I) a dificuldade de aceitação dos artigos por parte das revistas, (2) a crença de que as réplicas que confirmam a pesquisa original não contribuem significativamente para o conhecimento, o que as leva a não serem submetidas ou aceitas para publicações, (3) a hesitação em publicar resultados contraditórios aos do estudo original, porque podem ser fruto de erro na replicação, e (4) a crença de que pesquisas replicadas são consideradas inferiores por periódicos, pesquisadores e escolas.

Roch (2003, p.I65-167) afirma que "É mais fácil usar modelos externos do que construir teoria com base na realidade brasileira. É mais barato replicar do que desenvolver pesquisa original. É mais conveniente adaptar instrumentos de coleta e análise do que criar algo novo”. Nos levantamentos bibliográficos feitos, com base nas publicações científicas nacionais, não se identificaram evidências que confirmem tal afirmação. Ao contrário, confirmam-se os argumentos dos autores que declaram que replicar resultados de surveys já realizados tem recompensas relativamente baixas para custos substanciais (GLANTON, 2OIO; HAMERMESH, 2007; MADDEN; EASLEY; DUNN, 2005; EASLEY; MADDEN; DUNN, 2000). 


\section{REPLICAR É PARA QUEM?}

Reavaliar o lugar ocupado pelas repetições de pesquisas no âmbito acadêmico contribuiria com o surgimento de interesse por esse tipo de investigação, por parte de novos pesquisadores (EASLEY; MADDEN; DUNN, 2000). Parte da responsabilidade do baixo status das reproduções de estudos pode ser atribuída aos editores de revistas acadêmicas (UNCLES, 20II; MADDEN; EASLEY; DUNN, 2005), ao não reconhecerem a contribuição das replicações por elas não apresentarem novas ideias ou métodos. Editores proclamam a importância de repetir pesquisas, mas não as publicam (BERTHON et al., 2002).

Phillips e Pugh (1987) sugerem que essa prática de pesquisa seja utilizada por estudantes de mestrado. King (1995), por sua vez, recomenda a duplicação de pesquisas como inspiração de trabalhos de conclusão de curso para estudantes de graduação. Berndt e Oliveira (2005) sugerem a aplicação desta metodologia em trabalhos de estudantes de graduação e de pós-graduação lato e stricto- sensu. Todos estes autores concordam com a hipótese de que a validação do procedimento seja um meio de estimular a propagação do conhecimento metodológico e uma forma de assegurar a continuidade das investigações (MORRISON; MATUSZEK; SELF, 2OIO; BURMAN; REED; ALM, 20IO; BERNDT; OLIVEIRA, 2005; BERTHON et al., 2002; EASLEY; MADDEN; DUNN, 2000; KING, I995; PHILLIPS; PUGH, I987).

Portanto, de acordo com Easley, Madden e Dunn (2000), como modo de reafirmar o papel das replicações, os editores e revisores de artigos acadêmicos devem estimular a submissão dessas pesquisas às suas revistas e aos congressos acadêmicos. Outra iniciativa é a de que os revisores incentivem os trabalhos originais a disponibilizarem seus dados, de modo que estes possam ser repetidos. Tal argumento é chamado por King (1995) de "padrão de replicação". 


\section{CONSIDERAÇÕES FINAIS}

Conforme afirmou-se anteriormente, pesquisas quantitativas em Administração de Empresas não costumam ser foco de replicações (MORRISON; MATUSZEK; SELF, 20Io). Este fato deve-se a uma série de motivos, os quais já foram expostos acima. Também já foram expostas as razões pelas quais a repetição de pesquisas, especialmente aquelas que propõem novos constructos teóricos, é algo importante. Por ora, fazem-se algumas recomendações, com vistas ao aumento da prática da replicação.

Editores, sob o ponto de vista científico, poderiam promover a publicação de replicações, de modo a aumentar a confiabilidade dos resultados dos levantamentos de dados advindos de surveys das pesquisas acadêmicas (UNCLES, 2OII; HUNTER, 20OI).

Como política de publicação de artigos quantitativos, é recomendável que editores de periódicos e membros de universidades disponibilizem os dados utilizados nos estudos para a realização de replicações. Assim, os dados originais, as análises realizadas e os programas utilizados estariam acessíveis para verificação. Cabe salientar que informações confidenciais, como o nome dos respondentes, deveriam ser suprimidas (MORRISON; MATUSZEK; SELF, 20IO; EVANSCHITZKY et al., 2007; KING, I995).

Para que uma pesquisa possa ser repetida, recomenda-se que os autores registrem os padrões metodológicos, além de explicitarem, em artigos, livros ou dissertações, qual foi o processo de coleta e análise de dados que utilizaram. Isso demonstraria comprometimento com desenvolvimentos futuros do tópico pesquisado (VELUDO-DE-OLIVEIRA; OLIVEIRA, 2OI2; KING, 1995). Artigos com esta documentação foram duas vezes mais citados quando comparados aos artigos que não apresentaram tais registros, segundo a pesquisa de Evanschitzky e Armstrong (2009). Madden, Easley e Dunn (2005), em pesquisa comparativa de editores de revistas das ciências naturais e sociais, concluíram que nas ciências naturais a prática de replicações é geralmente aceita como parte integrante das pesquisas. Nas ciências sociais, por outro lado, os editores se mostram menos entusiasmados com a adoção da prática. 
"Erros inadvertidos em artigos publicados em journals econômicos são comuns e não têm ocorrência rara", afirmam Dewald, Thursby e Anderson (1986, p. 588 - tradução nossa). A contundente conclusão dos autores, após a condução de um projeto de replicação, foi a de que havia documentação suficiente para replicar apenas 10\% de um total de 150 pesquisas. No caso de surveys, a disponibilidade da documentação, além do registro adequado dos procedimentos metodológicos são aspectos necessários para que se repita a pesquisa original.

É amplamente aceita na literatura a afirmação de que não se replica suficientemente (TSANG; KWAN, 1999), e há indícios de que a postura dos editores de revistas científicas contribua para esta realidade (MADDEN; EASLEY; DUNN, 2005).

Identifica-se uma exceção notável com relação à atitude dos editores na revista Public Finance Review (2010), a qual convocou artigos de replicação (A Call for Replications Studies). A convocação por replicações foi justificada: a) pela crença de que journals devem publicar resultados de replicações (favoráveis ou desfavoráveis); (b) pelo fato de os softwares econométricos possuírem bugs; e (c) por pesquisas replicadas serem exceções e não a regra (BURMAN; REED; ALM, 2OIO).

No Brasil, da mesma maneira, as evidências sugerem que não se replica o suficiente nos estudos em Administração de Empresas. Os trabalhos analisados nos periódicos nacionais, que utilizaram a prática, lograram contribuir para a evolução do conhecimento científico, ainda que sua incidência seja extremamente baixa. Como reflexão, questiona-se, ainda, se todos os autores que replicaram trabalhos identificaram a prática em seus estudos.

O presente ensaio se presta a debater a importância da replicação, reflexão igualmente escassa na comunidade acadêmica nacional. A baixa incidência de replicação nacional nas pesquisas de metodologia quantitativas abre amplas oportunidades de utilização dessa prática para a construção do conhecimento. Isso ocorre especificamente entre as pesquisas do tipo survey, com a verificação da validade de estudos já realizados, e com a 
possibilidade que se abre para a realização de meta-análises, além de outros desdobramentos.

Da mesma forma, incentivam-se os editores de revistas brasileiras a estimularem a submissão de investigações com a utilização desta prática, como inspiração e fundamento para outros trabalhos. 


\section{REFERÊNCIAS}

ALRECK, P. L.; SETTLE, R. B. The survey research handbook, MacGraw-Hill Higher Education 3.ed., 2004.

BERNDT, A.; OLIVEIRA, L. A Construção do Saber Administrativo por meio de Replicagens em Pesquisas por Levantamento (Survey). Revista ANGRAD, v. 6, n. 3, p. 9 $-26,2005$.

BERTHON, P.; PITT, L.; EWING, M.; CARR, C. L. Potential Research Space in MIS: A Framework for Envisioning and Evaluating Research Replication, Extension and Generation. Information Systems Research, v. 13, n. 4, p. 416 - 427, 2002.

BIDO, D.; SOUZA, C.; SILVA, D.; GODOY, A.; TORRES, R. Qualidade dos Relatos dos Procedimentos Metodológicos em Periódicos Nacionais na Área de Administração de Empresas: o caso da modelagem de equações estruturais nos periódicos nacionais entre 2001 e 2010. O\&S Salvador, v. 19, n. 60, p. 125 - 144, 2012.

BURMAN, L.; REED, R.; ALM, J. A Call for Replications. Public Finance Review, v. 38, n. 6, p. 787- 793, 2010.

CAPPELLE, M.; BRITO M.; MELO, M.; VASCONCELOS, K. A produção científica sobre gênero na Administração: uma meta-análise. In: ENCONTRO NACIONAL DE PÓS-GRADUAÇÃO E PESQUISA EM ADMINISTRAÇÃO, 30., 2006, Salvador. Anais... 2006.

COLLIS, J.; HUSSEY, R. Business Research: a practical guide for undergraduate and postgraduate students. Palgrave Macmillan, v. 9, 1997.

COOPER, D.R.; SCHINDLER, P.S. Business Research Methods, MacGraw-Hill International Edition, 2008.

CRESWELL, J. W. Research Design: Qualitative and Quantitative Approaches, Thousand Oaks: Sage, 1994.

DARLEY, W. K. Status of Replication Studies in Marketing: a validation and extension. The Marketing Management Journal, v. 10, n. 2, p. 121-132, 2000.

DEWALD, W.; THURSBY, J.; ANDERSON, R. Replication in Empirical Economics: The Journal of Money, Credit and Banking Project. American Economic Review, v. 78, n. 1162- 1163. 1986.

EASLEY, R. W.; MADDEN, C. S.; DUNN, M. G. Conducting Marketing Science: The Role of Replication in the Research Process. Journal of Business Research, v. 48, n. 1, p. 83-92, 2000.

EDEN, D. Replication, Meta-Analysis, Scientific Progress, and AMJ's Publication Policy. Academy of Management Journal, v. 45, n.5, p. 841-846, 2002.

EVANSCHITZKY, H.; ARMSTRONG, J. S. Replications of Forecasting Research. International Journal of Forecasting. p. 1- 12, 2009. 
EVANSCHITZKY, H.; BAUMGARTH, C.; HUBBARD, R.; ARMSTRONG, J. S.; Replication research's disturbing trend. Journal of Business Research, v. 60, p. 411- 415. 2007.

FENSTERSEIFER, J. E.; SAUL, N. Investimentos de capital nas grandes empresas. Revista de Administração, v. 28, n. 3, p. 3-12, 1993.

GLANTON, P. Report on the American Economic Review Data Availability Compliance Project Vanderbilt University, p. 1-12, 2010.

HAMERMESH, D. Viewpoint: Replication in Economics. Canadian Journal of Economics, v. 40, n. 3, p. $715-733,2007$.

HENDRICK, C. Replication, Strict Replications, and Conceptual Replications: are they important? In: NEWLIEP, J.W. (Ed.). Replication Research in the Social Sciences. Newburry Park, Sage Publications, 1991, p. 41-49.

HUBBARD, R.; VETTER, D.; LITTLE, E.; Replication in Strategic Management: Scientific Testing for Validity, Generalizability, and Usefulness. Strategic Management Journal, v. 19, p. 243-254, 1998.

HUNTER, J. The Desperate Need for Replications. Journal of Consumer Research, v. 28, p. 149-158, 2001.

KING, G. Replication, Replication. Political Science \& Politics, Harvard University, p. 444-452, 1995.

MADDEN, C. S.; EASLEY, R.W.; DUNN, M. G. How Editors View Replication Research. Journal of Advertising, v. 29, n. 4, 2005.

MARCHETTI, R.; PRADO, P. H. M. Avaliação da satisfação do consumidor utilizando o método de equações estruturais: um modelo aplicado ao setor elétrico brasileiro. Revista de Administração Contemporânea, Curitiba, v. 8, n. 4, 2004.

MARTIGNAGO, G.; ALPERSTEDT, G. A Pesquisa em Gestão Internacional no Brasil entre 2001 e 2010: Um estudo a partir dos Anais do Enanpad, In: ENCONTRO NACIONAL DE PÓS-GRADUAÇÃO E PESQUISA EM ADMINISTRAÇÃO, 35, Rio de Janeiro, Anais..., 2011.

MORENO, A. B.; FAERSTEIN, E.; WERNECK, G. L.; LOPES, C. S.; CHOR, D. Propriedades psicométricas do Instrumento Abreviado de Avaliação de Qualidade de Vida da Organização Mundial da Saúde no Estudo Pró-Saúde. Caderno de Saúde Pública, 2006.

MORRISON, R; MATUSZEK, T.; SELF, D. Preparing a Replication or Update Study in the Business Disciplines. European Journal of Scientific Research, v. 47, n.2, p. 278-287, 2010. NEVES, J. A identidade e a focagem dos empregados de restaurantes de Fortaleza. Revista de Admnistração, São Paulo, v. 42, n.4, p. 443-453, 2007.

NEVES, J. A. D.; COSTA, A. M. Fatores de localização de postos de combustíveis em Fortaleza. RAC - Revista de Administração Contemporânea, v. 12, edição especial, 2008. 
PHILLIPS, E.; PUGH, D. S. How to Get a PHD: a handbook for students and their supervisors, Open University Press, Buckingham, Philadelphia, 2.ed, 1987.

PRATES, A. A. P. Redes sociais em comunidades de baixa renda: os efeitos diferenciais dos laços fracos e dos laços fortes. Revistade Admnistração Pública, Rio de Janeiro, v. 43, n. 5, 2009.

POMERANCE, E.; ZIELSKE, H. How frequently should you advertise? Media/Scope, 1959.

RIEDL, M. Paradigmas Teóricos de Estrutura e Mudança Social. Santa Cruz do Sul: UNISC, 2007.

ROCH, S. Quem Responde pelo Desempenho Limitado da Produção Científica em Administração no Brasil? Organizações e Sociedade, v. 10, n. 28, p. 165-167, 2003.

RODRIGUES, A.; ASSMAR, E. M. L. Influência social, atribuição de causalidade e julgamentos de responsabilidade e justiça. Psicologia, Reflexão e Crítica, v. 16, n. 1, 2003.

SHENG, H. H.; SAITO, R. Análise de Métodos de Replicação: o Caso Ibovespa. Revista de Administração de Empresas, v. 42, n. 2, 2002.

SINGH, K.; ANG, S.; LEONG, S. Increasing Replication for Knowledge Accumulation. Strategy Research. Journal of Management, v. 29, n. 4, p. 533-549, 2003.

SIMON, J. What Do Zielske’s Real Data Really Show About Pulsing? Journal of Marketing Research, v. 16, n.1, p.415- 420, 1979.

TENÓRIO, F. Gestão Social: Uma replica. Revista ADM. MADE, v. 13, n. 2, p.1-4, 2009.

TONCAR, M. F.; MUNCH, J. M. Meaningful replication: when is a replication no longer a replication? A rejoinder to Stella and Adam. Journal of Marketing Theorye Practice, v. 18, n. 1, p. 71-80, 2010.

TSANG, E.; KWAN, K. Replication and Theory Development in Organizational Science: a Critical Realistic Perspective. Academy of Management Review, v. 24, n. 4, p. $759-780,1999$.

UNCLES, M. Publishing Replications in Marketing. International Journal of Market Research, v. 53, n. 5, 2011.

VELUDO-DE-OLIVEIRA, T. M.; OLIVEIRA, B. Diretrizes para a adequação metodológica e integridade da pesquisa em Administração. RAD, v.14, n.1, 2012.

YASSUDA, M. S.; BATISTONI, S. S. T.; FORTES, A. G.; NERI, A. L. Treino de memória no idoso saudável: benefícios e mecanismos. Psicologia: Reflexão e Crítica, v. 19, n. 3, p. 470-481, 2006. 


\section{DADOS DOS AUTORES}

\section{MARIA LAURA FERRANTY MAC LENNAN` ferranty@hotmail.com} Mestre em Administração de Empresas pela ESPM

Instituição de vinculação: Escola Superior de Propaganda e Marketing São Paulo/SP - Brasil

Áreas de interesse em pesquisa: Estratégia empresarial, metodologia de pesquisa e internacionalização de empresas.

${ }^{\star}$ Rua Dr. Álvaro Alvim, 123 Vila Mariana São Paulo/SP 04018-010

\section{ILAN AVRICHIR iavrichir@espm.br}

Doutor em Administração de empresas pela EAESP/FGV

Instituição de vinculação: Escola Superior de Propaganda e Marketing São Paulo/SP - Brasil

Áreas de interesse em pesquisa: competitividade de empresas brasileiras, cultura organizacional, metodologia de pesquisa, empreendedorismo internacional e estudo de caso. 